\title{
Reinserción laboral de personas que cumplen condena en libertad: un acercamiento desde los encargados de los Programas de Intermediación Laboral en el Sistema Abierto de Gendarmería de Chile
}

Reintegration into labor market of people serving their sentence in probation: an approach from responsibles of job placement programs in probation system of Gendarmería de Chile

Daniela Andrea Ibacache Ramírez. Asistente social. Licenciada en Trabajo Social. Magíster en Trabajo Social y Organizaciones Pontificia Universidad Católica de Chile.Email: daibacache@uc.cl / dibacacheramirez@gmail.com

\begin{abstract}
Resumen
El Programa de Intermediación Laboral en el Sistema Abierto de Gendarmería de Chile interviene a personas que cumplen condena en libertad, apoyando el proceso de reinserción social mediante la nivelación escolar, capacitación y colocación laboral. Un estudio que evaluó el impacto del programa evidenció una disminución en las cifras de reinserción laboral, lo que generó preocupación institucional por conocer y mejorar los procesos de intervención. Bajo este contexto, se realizó una investigación diagnóstica a los encargados de programa a nivel nacional, con la finalidad de conocer cómo se estaban implementando los talleres de apresto laboral y cuáles eran las necesidades sociolaborales de los usuarios. Utilizando una metodología cualitativa se recolectaron los datos, los cuales fueron analizados con la teoría fundamentada. Los resultados dieron cuenta de una ausencia de lineamientos generales de intervención, las principales necesidades sociolaborales de los usuarios. Éstas se relacionaban con el déficit de habilidades de comunicacionales, habilidades de resolución de conflicto y competencias laborales.
\end{abstract}

Palabras Claves. Infractor de ley, Reinserción social, Reinserción laboral, Gendarmería de Chile.

\begin{abstract}
The job placement program in probation system of "Gendarmería de Chile" intervene to people serving their sentence in freedom, supporting the reintegration into society process by means of scholar leveling, training, and labor placement. A research which assessed the impact of the program displayed a decrease of reintegration into labor market figures. This produced institutional concern about knowing and improving intervention processes. Under this context, it was conducted a national diagnostic research to the program responsibles, with the purpose to know how the job placement preparation workshops were held, and which were the social-labor needs of the users. Using a qualitative methodology, the data were gathered and analyzed under grounded theory. The results accounted for an absence of general guidelines of intervention; and the main users' social-labor needs. These needs were related to the communicational skill, conflict resolution skills, labor competences deficiency.
\end{abstract}

Key words. Offender, Reintegration into Society, Reintegration into Labor Market, Gendarmería de Chile. 


\section{Introducción}

Una de las principales preocupaciones para las autoridades chilenas es la de reinsertar socialmente a aquellas personas que han cometido algún delito. Esto debido al aumento en las tasas de reincidencia delictual, que para quienes cumplieron condena en el sistema cerrado (cárcel) alcanzó un 50,5\% y para quienes lo hicieron el sistema abierto (en libertad) alcanzó un 27,7\% (Fábrega, Morales, Muñoz \& Welsch, 2012), lo que ha generado una interrogante sobre cuáles son las intervenciones que se están ejecutando para reinsertar a este segmento de población.

Las personas que cumplen condena en el sistema abierto lo hacen en los Centros de Reinserción Social (en adelante CRS) dependientes de Gendarmería de Chile, organismos que actualmente atienden a 51.541 usuarios, lo que equivale a un 53\% del total de la población penal chilena (Gendarmería, 2014). Dentro de su oferta programática, los CRS otorgan a los usuarios programas de apoyo para los procesos de reinserción laboral y social. Uno de ellos es el Programa de Intermediación Laboral en el Sistema Abierto (en adelante PILSA), el cual mediante la nivelación escolar, capacitación en algún oficio y colocación laboral, busca fortalecer habilidades y conocimientos sociolaborales, facilitando el proceso de reinserción laboral y social de los usuarios.

La investigación surge a partir de una necesidad institucional del Departamento de Reinserción Social en el Sistema Abierto (en adelante DRSSA) de Gendarmería de Chile, la cual se relaciona con el diseño de un instrumento que permita mejorar los resultados de intervención del PILSA. Por ello, esta se enfocó en la realización de un diagnóstico a los encargados del programa con finalidad de conocer cómo se estaban implementando los talleres de apresto laboral (fase de inicio en el proceso de intervención del programa que tiene por objetivo preparar a los usuarios con las herramientas necesarias para enfrentar el mundo laboral), así como también conocer cuáles eran las necesidades sociolaborales de los usuarios. Con los resultados obtenidos en el diagnóstico, se realizó una propuesta de intervención con la finalidad de facilitar los procesos de intervención e idealmente permitir que los usuarios se reinserten a la sociedad.

El trabajo es considerado como herramienta socialmente validada, por la cual el ser humano es reconocido y valorado en la comunidad (Honneth, 2009). La disponibilidad de un empleo ha sido reconocido como un relevante mecanismo para generar cambios en la conducta de quienes han co- metido delitos, así como también para alcanzar la reinserción social (Alós, Martín, Miguélez \& Gibert, 2009; Espinoza, Ferrada, Navarro \& Valenzuela, 2012). De esta forma, Atkin \& Armstrong (2013) sostienen que las herramientas con las que cuenta un individuo antes de enfrentarse a un trabajo son de real importancia para el mantenimiento de éste, es decir, no sólo la biografía laboral es importante, sino que también las competencias laborales con las que el sujeto cuente. A partir de ello, Cid \& Martí (2011) agregan que el trabajo parece ser una condición fundamental para encarar una transición exitosa en la vida en libertad.

\section{Discusión bibliográfica}

La delincuencia es un tema que nos afecta a todos, pues no sólo afecta la conducta del individuo infractor de ley, sino que también trasciende a la sociedad (Fábrega et al., 2012). Las diversas maneras de enfrentarla no sólo se reducen a aquellas que de manera punitiva (represiva-policial) se orientan por la utilización de la privación de libertad como medio para la resolución de conflicto, sino que también existen otras (penas sustitutivas a la reclusión) que permiten al individuo cumplir su condena en libertad.

Conforme a lo anterior, la reinserción social aparece como una de las vías para combatir la delincuencia (Oliveri, 2011). En este sentido, podemos definirla como un proceso de comunicación e interacción entre la sociedad y el infractor de ley, visualizándolo como sujeto de derecho que reclama en su comunidad atención para desarrollar fines trascendentales de su vida, mereciendo una oportunidad de participar en ella y cuya integración es responsabilidad de todos (Espinoza \& Viano, 2008). En otras palabras, la reinserción social no sólo está en manos del infractor de ley o del organismo que lo interviene, sino que también el rol de la sociedad en este proceso conlleva a responsabilizarse por sus mecanismos de exclusión (Olate, 2007; Baratta, 2002 citado en Añez, Han, Morales \& Párraga, 2008).

Uno de los exponentes que plantea la importancia que mantiene la sociedad en términos de reconocimiento, valoración social y autorrealización de los sujetos es Axel Honneth en su Teoría del Reconocimiento (1997), el cual sostiene que el reconocimiento en la esfera de la sociedad civil se gesta a partir de la valoración social de una persona, y ésta se mide por las aportaciones que ella hace a la sociedad en forma de un trabajo formalmente organizado (Honneth, 2009). La oportunidad de 
realizar un trabajo económicamente remunerado y socialmente regulado va unido a la adquisición de una forma de reconocimiento y valoración social. De esta forma, se concibe a la sociedad civil como uno de los elementos relevantes para la transformación y reinserción social de estas personas.

De acuerdo a lo anterior, el trabajo se transforma en un elemento clave para alcanzar la reinserción laboral y social de quienes cumplen condena en libertad. Para Ibañez (2012), el trabajo formal tiene la ventaja de producir inserción social de personas en situación de vulnerabilidad, puesto que se hacen parte de un sistema que anteriormente no los incluía, generando en ellos un sentimiento de pertenencia. El trabajo es considerado por la CEPAL (2009) como un derecho humano fundamental para la integración social y espacio de participación. Es considerada como una herramienta básica para alterar el comportamiento de un infractor de ley en pro de su reinserción social, ya que permite al sujeto poseer una visión de cambio por medio de una actividad socialmente validada (Alós, Martín, Miguélez, \& Gibert, 2009).

Como parte del proceso de concientización de la sociedad en los procesos de reinserción social, se modificó la Ley 18.216, sustituyéndose en el año 2013 por la Ley 20.603, la cual amplió las penas sustitutivas a la reclusión; Remisión condicional, Reclusión parcial, Libertad vigilada, Libertad vigilada intensiva, Expulsión de extranjeros y Prestación de servicios en beneficio de la comunidad, permitiendo a los usuarios cumplir condena en libertad, evitando los efectos nocivos que trae consigo la privación de libertad, así como también permite un mejor acceso al proceso de reinserción social (Visher, Winterfield \& Coggeshall, 2005; Kalinsky, 2012).

La evidencia internacional y nacional ha demostrado que los programas de reinserción laboral penitenciaria que se enfocan en desarrollar habilidades sociales, nivelar estudios, capacitar y colocar laboralmente a los usuarios, aportan al logro de la reinserción laboral y reducen las tasas de reincidencia (McMurran \& McCulloch, 2007; Luong \& Wormith, 2011; Tarlow, 2008). La efectividad de estos programas está relacionada con la adecuada detección de necesidades y habilidades sociolaborales y las causas por la que los programas laborales fracasan tienen relación con que éstos no dan respuesta a las necesidades de los usuarios ni a sus motivaciones (Andrews, Bonta \& Wormith, 2006; Fundación Paz Ciudadana, 2010; Yahner, Zweig \& RedCross, 2010).
Acevedo, Guzmán, Pantoja \& Weinborn (2009) sostienen que el desarrollo y potenciación de competencias cognitivas, psicosociales, relacionales y laborales en las personas que cumplen condena son un medio que facilita la integración a espacios sociales y laborales. Una competencia laboral es definida como la construcción social de aprendizajes significativos y útiles para el desempeño productivo en una situación real de trabajo, que se obtiene no sólo a través de la instrucción, sino también mediante el aprendizaje por experiencia en situaciones concretas de trabajo (Gallart \& Jacinto, 1995; Jaraba, Romero \& Ruiz, 2005). Por ello, las intervenciones sociales en materia de reinserción laboral requieren potenciar las competencias y habilidades sociolaborales de sus usuarios, detectando adecuadamente sus necesidades y motivaciones.

El Modelo de Riesgo-Necesidad-Capacidad de Responder (Risk-Need-Responsivity) resulta adecuado para diagnosticar y potenciar habilidades laborales y sociales, donde la evidencia demuestra que aportaría a la disminución de los índices de reincidencia delictual y a la reinserción social (Hollin \& Palmer, 2009; Andrews y otros, 2012). Este modelo supone que mientras mayor es el riesgo de reincidencia del sujeto, las intervenciones deben ser más intensas, abordando sus necesidades criminógenas, adecuando la intervención al estilo de aprendizaje del usuario, considerando fortalezas o competencias, factores que tienen el potencial de facilitar u obstaculizar la intervención.

De igual manera, la literatura revisada otorga relevancia al Modelo Transteórico de Motivación para el Cambio, el cual supone que la motivación para el cambio no ocurre de manera espontánea, sino que sigue un patrón de etapas claramente definidas (DiClemente, Prochaska \& Norcross, 1992). Considerando las motivaciones y necesidades del usuario, el diseño de las intervenciones sociales en materia de reinserción laboral debe enfocarse en fomentar la motivación, potenciar conocimientos y habilidades sociolaborales para que se entreguen las herramientas necesarias para acceder y permanecer en un trabajo. Un estudio sobre reinserción laboral en el medio libre afirma que la reincidencia de aquellos usuarios que participan o participaron de un programa laboral es menor que la de aquellos que no lo hicieron (González \& Ramírez, 2008).

Diversos autores afirman que las herramientas con las que cuenta el individuo antes de ingresar a un trabajo son de real importancia para el mantenimiento en este. Por ello, el desarrollo o potenciación de las habilidades sociolaborales que poseen o de las que 
carecen los usuarios que ingresan al programa median el éxito o permanencia en una capacitación o colocación laboral (Atkin \& Armstrong, 2013; Cid \& Martí, 2013).

Un estudio que evaluó los resultados del PILSA a nivel nacional demostró que en el periodo 2008-2011 disminuyeron los capacitados colocados laboralmente de un $42,2 \%$ a un $33,5 \%$, hubo un aumento en la deserción del programa de un 9,4\% a un 13\%, los usuarios con empleo dependiente que percibían un salario mínimo cayeron de un 54\% a un 38\% y los usuarios que permanecieron en el trabajo a los dos meses de colocados disminuyeron de un $82 \%$ a un 59\% (Espinoza, Ferrada, Navarro \& Valenzuela, 2012). Estas cifras evidencian la dificultad de empleabilidad que posee la población infractora, sobre todo considerando la falta de competencias sociolaborales, certificación educativa y experiencia laboral, lo cual se ha presentado como un real desafío para los coordinadores de los PILSA, ya que además de realizar el control de la pena, conjuntamente deben facilitar las herramientas adecuadas para que los usuarios ingresen y permanezcan en un trabajo, y de esta forma logren su reinserción laboral. En base a estos antecedentes, surge la interrogante sobre ¿cómo implementan los talleres de apresto laboral los encargados de los PILSA de Gendarmería de Chile?

A partir de la literatura revisada y los antecedentes empíricos expuestos, la reinserción laboral es considerada como una vía para alcanzar la reinserción social de aquellas personas que han infringido la ley penal. Ésta debe ser reinterpretada y reconstruida sobre una base diferente, donde la sociedad sea partícipe en este proceso, por lo que la reinserción de un infractor de ley signifique, antes que una transformación separada de su contexto, la transformación de la sociedad que reasuma parte de sus problemas y conflictos que se encuentran segregados en la población que cumple condena.

\section{Metodología}

La investigación tuvo como objetivo central describir la implementación de los talleres de apresto laboral que realizan los encargados de programa y conocer las necesidades sociolaborales de los usuarios del PILSA. Para el logro del objetivo se utilizó una metodología cualitativa con un diseño de investigación descriptivo. La cual permitió conocer, comprender y describir el fenómeno social, a partir de las propias vivencias de los sujetos de estudio (Krause, 1995).
Los participantes fueron seleccionados mediante un muestreo estratégico, característico del diseño cualitativo, donde no se persiguió la representatividad de la muestra, sino la generatividad del caso, es decir las posibilidades de dar cuenta de modos de comprender y construir versiones sobre realidades más plurales (Flick, 2004). Con base en ello, se seleccionaron informantes claves, quienes permitieron acercarse y comprender en profundidad la realidad social de los PILSA. A partir de lo anterior, se definieron criterios de inclusión para la selección de los participantes, los cuales tenían relación con; poseer un año de experiencia en el PILSA, haber desarrollado talleres de apresto laboral y pertenecer a los siete programas que atienden a una mayor cantidad de usuarios a nivel nacional, los que corresponderían a los programas de Santiago, Valparaíso, Linares, Antofagasta, Chillán, Valdivia y Puerto Montt. Por tanto, la muestra finalmente quedó conformada por siete profesionales, encargados de programas correspondientes a las regiones antes mencionadas. Se utilizaron dos técnicas para la recolección de datos, entrevistas estructuradas no presenciales (enviadas por vía email a los encargados de los programas de Antofagasta, Chillán, Valdivia y Puerto Montt) y entrevistas semiestructuradas presenciales (realizadas a los encargados de programa de Santiago, Valparaíso y Linares), las cuales permitieron conocer y comprender las perspectivas que tienen los encargados de programa respecto de sus experiencias o situaciones con los talleres de apresto laboral, expresadas por sus propias palabras (Taylor \& Bodgan, 1987). Se utilizó una pauta de doce preguntas para ambos instrumentos. Los participantes fueron contactados uno a uno vía email e invitados a participar de la investigación. Cada uno recibió información respecto a la confidencialindad de la información y los objetivos generales del estudio. Una vez que estos aceptaron participar, se enviaron y realizaron las entrevistas correspondientes a los participantes seleccionados, las que fueron recepcionadas y ejecutadas en su totalidad, no presentando inconvenientes para su posterior análisis. Los datos recolectados fueron analizados de acuerdo a la Teoría Fundamentada o Grounded Theory (Corbin \& Strauss, 2002), la que permitió indagar inductivamente en el fenómeno, accediendo a los significados que los sujetos otorgaron a su discurso. Luego de realizar las entrevistas a los encargados de los PILSA y mediante los datos arrojados en cada una de ellas, se dio lugar a la creación de tipologías descriptivas que se desarrollaron por medio de una "codificación abierta", extrayendo conceptos claves 
respecto del fenómeno foco de estudio, que permitieron articular el material analíticamente, a través de una conceptualización y categorización de los datos recogidos

\section{Resultados}

Con base en el análisis realizado a partir de la teoría fundamentada, se obtuvieron tres categorías descriptivas, las que se discutirán considerando los aportes de estudios, experiencias y teorías generadas entorno a ellas.

La primera de ellas se relaciona con el Diseño y procedimiento de los talleres de apresto laboral, donde los encargados de programa sostienen que no existen lineamientos generales de intervención para poder ejecutar el taller, el número de sesiones es variable según la disponibilidad de tiempo del encargado o el número de participantes del taller. A su vez, agregan que los objetivos de intervención debieran ser más realistas o alcanzables, ya que se generan frustraciones y desmotivaciones de los usuarios al no poder alcanzarlos. "...Yo creo que el trabajo que hace el programa es muy interesante y coayudador de la libertad vigilada... Por eso es muy importante ser cuidadosos con la promesa del programa laboral con los penados, que no se generen tremendas frustraciones cuando se interviene...." (E2, P26-27).

Los talleres son ejecutados con diversos materiales pedagógicos que no se ajustan a las necesidades usuarias y son insuficientes para dar cumplimiento a los objetivos organizacionales. "Actualmente nos mantenemos a la espera de módulos de apresto laboral que serán enviados desde el nivel central, no obstante se interviene con material trabajado en años anteriores en este CRS y que tiene relación a módulos de apresto dirigido a trabajadores dependiente e independientes" (E6, P10).

Miller \& Rollnick (2002) concuerdan con lo argumentado por los encargados. Plantean que los objetivos de intervención deben ser realistas, fáciles de alcanzar, valorados por el usuarios, con una enunciación concreta y medibles, lo que permite visualizar sus avances e impulsa una intervención satisfactoria.

De la misma forma, Bermejo \& Martínez (2006) afirman que para que un objetivo de intervención provoque resultados positivos debe ser interiorizado y asumido como propio por el usuario y no autoimpuesto.

Day, Bryan, Davey \& Casey (2006) realizaron un estudio donde evaluaron diversos programas de reinserción laboral, los cuales arrojaron que los formatos más efectivos son aquellos intensivos, a cargo de un equipo especializado que detecta necesidades y ajusta la intervención al usuario, basado en el modelo Risk-Need-Responsivity (RNR) (Andrews, Bonta \& Wormith, 2006). A su vez, el estudio demostró que usuarios que se mantienen en estos programas logran reducir hasta en un 40\% los índices de reincidencia delictual.

La segunda categoría hace referencia al Perfil del Usuario que asiste a los talleres de apresto laboral. Los hallazgos dan cuenta de la existencia de dos perfiles de usuarios; usuarios jóvenes menores de 35 años que no poseen un proyecto vital ni laboral claramente definido, poseen mayor riesgo a reincidir, y usuarios adultos, mayores de 36 años, quienes mantienen ideales y metas laborales elaboradas y ven en el trabajo y el programa una oportunidad para alejarse de la delincuencia.

"Los usuarios tienen trayectoria laboral muy precaria... la mayoría no tiene experiencia laboral, y yo les pregunto en qué quieren trabajar y me dicen en cualquier cosa, esas son personas hasta 35 años. Ya los otros, es gente más madura, más dispuesto al sacrificio, estar viniendo dos meses a clases, vienen más dispuesto..." (E2, P21).

Ambos perfiles de usuarios se caracterizan por poseer una precaria experiencia laboral, bajos niveles de escolaridad y certificación laboral. Las principales necesidades sociolaborales que los encargados de programa visualizan en los usuarios se relacionan con la falta de habilidades comunicacionales, resolución de conflictos y competencias laborales. Su principal motivación es conseguir un trabajo remunerado.

"Una motivación que tenga que ver con una concepción de trabajo más abstracta, así como de realización personal, de trascendencia, no, por lo menos yo nunca he observado eso. O de sentir que está prestando un servicio al otro, a través de lo que pueda estar haciendo, no. Acá la necesidad concreta que tenía que ver con satisfacer un requerimiento de la libertad vigilada que era estar trabajando o capacitándose, o bien porque hay una necesidad material que es trabajar para obtener recursos para su familia o para sí mismo" (E1, P45).

Con base en lo anterior, Ward \& Maruna (2007) sostienen que la mayoría de los infractores de ley abandonan su conducta delictual antes de la edad adulta, el 85\% desistiría del delito alrededor de los 35 años, antecedentes que se relacionan con los hallazgos de la investigación. Fundación Paz Ciudadana (2010) afirma que la colocación laboral sería más efectiva para reducir la reincidencia en 
infractores adultos que en jóvenes, en cambio en la población de infractores jóvenes sería más positivo fomentar la capacitación o educación. Asímismo, Howard, Clark \& Garnham (2006) afirman que los infractores de ley están entre las personas menos educadas y capacitadas de la sociedad, explicando que el déficit de habilidades limita las oportunidades para obtener un trabajo, situación que se agrava con las exigencias de sociedad y el mercado laboral. Laub \& Sampson (2001) afirman que episodios de desempleo llevan a una tasa más elevada de delitos. Quienes poseen antecedentes penales, esperan encontrar empleo rápidamente, expectativas que no son realistas dada su limitada experiencia laboral y falta de habilidades sociales, desventajas que constituyen el mayor obstáculo para encontrar un buen trabajo, por lo que la inestabilidad laboral se traduce en reincidencia. De igual forma, Howard, Clark \& Garnham (2006) sostienen -al igual que lo evidenciado por los encargados de programa- que los usuarios poseen habilidades de afrontamiento deficitarias, déficit cognitivos, déficit en el control de impulso, resolución de conflictos, pensamiento rígido e inflexible, los cuales si se intervienen disminuirían la reincidencia delictual y fomentarían la reinserción laboral. Por ello, Milkman \& Wanberg (2007) afirman que la intervención de infractores de ley debe enfocarse en el desarrollo de habilidades sociales, que involucren aprendizaje o fomento de habilidades de comunicación eficaz, habilidades interpersonales, resolución de conflictos y manejo de la agresividad.

Diversos autores plantean que una de las explicaciones del fracaso de los programas en el área laboral es que no se consideran las motivaciones intrínsecas ni extrínsecas de los usuarios, por ende el programa no da respuesta a las necesidades de este (Day et al., 2006; Atkin \& Armstrong, 2013; McMurran $\&$ McCulloch, 2007). En este contexto, Yahner, Zweig \& RedCross (2010) plantean que los talleres de apresto laboral deberían tener como incentivo la obtención de un puesto de trabajo, idea que se relaciona con lo planteado por los encargados, los que afirman que a los usuarios les motivaría mucho más que la participación en el taller finalizara con una colocación laboral.

Por ello, se hace necesario -y así dieron cuenta los encargados de programa- contar con un instrumento diagnóstico que les permita indagar en aquellas necesidades laborales y motivaciones de los usuarios, ya que a partir de ello es posible diseñar una intervención adecuada que sea capaz de dar cumpliento a los objetivos organizacionales.
Por último, la tercera categoría descriptiva hace referencia a las Expectativas para un futuro taller de apresto laboral, donde los encargados de programa coinciden y sugieren que el taller debe ser una instancia para conocer al usuario en profundidad, donde se pueda realizar un diagnóstico asertivo en relación con las habilidades que el usuario ya posee y desde allí comenzar a intervenir, es decir aceptar al usuario con sus características.

"Yo siempre he dicho el taller de apresto laboral, bueno tiene un objetivo como bien claro, pero ojalá este trabajo de reinserción sea integral, ayudar a descubrir a las personas en otro tipo de potencialidades, a lo mejor tiene habilidades para el arte, para el deporte, para lo social. Y a partir de ello comenzar a potenciar lo laboral. Para mí los talleres de apresto son un espacio para poder conocer a las personas, conocer sus potencialidades y ver cómo eso lo podemos conjugar con lo laboral" (E1, P51).

Bermejo \& Martínez (2006) plantean que los programas deben ser flexibles, adaptándose a la posición del usuario y sus estilos de aprendizaje. En la medida que las intervenciones se adapten a estas características se facilita el cumplimiento de sus objetivos. En este sentido, White \& Epston (2004) agregan que la persona es una cosa y sus problemas otros, es decir ésta es distinta al problema que le aqueja, por ello no debe estigmatizarse a la persona como un infractor de ley.

Ward \& Marshall (2007) sugieren que las intervenciones con infractores de ley debieran basarse en el autoconocimiento y el desarrollo de habilidades de acuerdo a sus intereses y capacidades. La construcción y reformulación de la identidad personal y social del individuo que está cumpliendo condena es uno de los primeros caminos para alcanzar la reinserción social (Acevedo, Guzmán, Pantoja \& Weinborn, 2009).

“... por eso te digo, que para mí es interesante la idea de un espacio de conocimiento. Porque ya esto es el usuario, esto es él o ella, con sus características, con sus defectos $y$ virtudes, con sus potencialidades y las que no, cómo no poder buscar una tarea o un trabajo que se adecue, que sea un poco más consistente o acorde a lo que esa persona es y... si la persona tiene la voluntad de reinventarse en algún área de su vida, primero que todo tiene que ser ésta de dejar el camino delictual" (E1, P49).

Diversos estudios dan cuenta de la efectividad de programas sociolaborales basados en Modelo de Riesgo-Necesidad-Capacidad de Respuesta y sostienen que disminuye considerablemente la reincidencia delictual, así como también demuestra efectos positivos en las habilidades de 
resolución de conflictos y comunicacionales. Las intervenciones deben centrarse en las necesidades criminológicas de los usuarios (fortalecimiento o desarrollo de factores de riesgo dinámicos; construcción de habilidades de manejo de conflicto, fomento del pensamiento prosocial, fomento de habilidades para el trabajo/estudio y fomento de actividades participativas), así como también debe coincidir con sus estilos de aprendizaje, aptitudes, habilidades y motivaciones (Acevedo, Guzmán, Pantoja \& Weinborn, 2009; Luong \& Wormith, 2011; Hollin \& Palmer, 2009).

Luego de realizar el análisis de las variables que describen la implementación de los talleres de apresto laboral y a partir de los antecedentes expuestos en la fase diagnóstica de la investigación, se representará de una manera gráfica el problema de investigación detectado, sus causas y consecuencias, para poder comprenderlo de manera expositiva, así como también se propondrá una solución a dicha problemática, Todo esto basado en Modelo de Problema y Modelo de Solución, planteado por Marcelo Didier (1986) en su libro Diseño y Evaluación de Programas Sociales, quien sostiene que exponer los resultados diagnóstico de investigación de esta manera facilita la comprensión de todas las variables involucradas en el problema y solución.

\section{MODELO DE PROBLEMA}

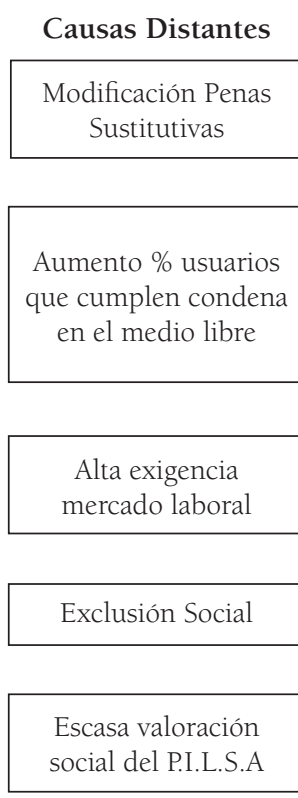

\section{Causas Directas}
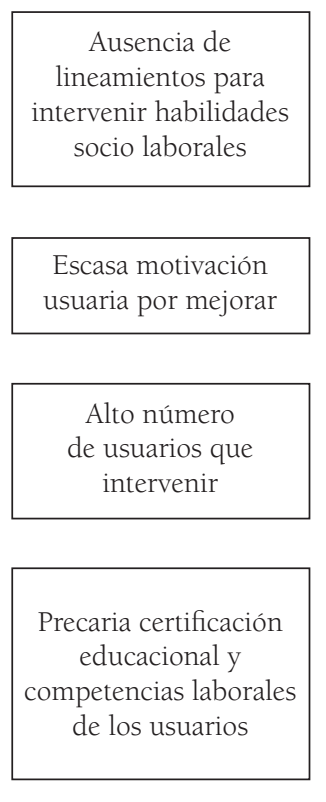

Escasos RRHH y apoyo pedagógico

Diversidad de concepción del Apresto Laboral

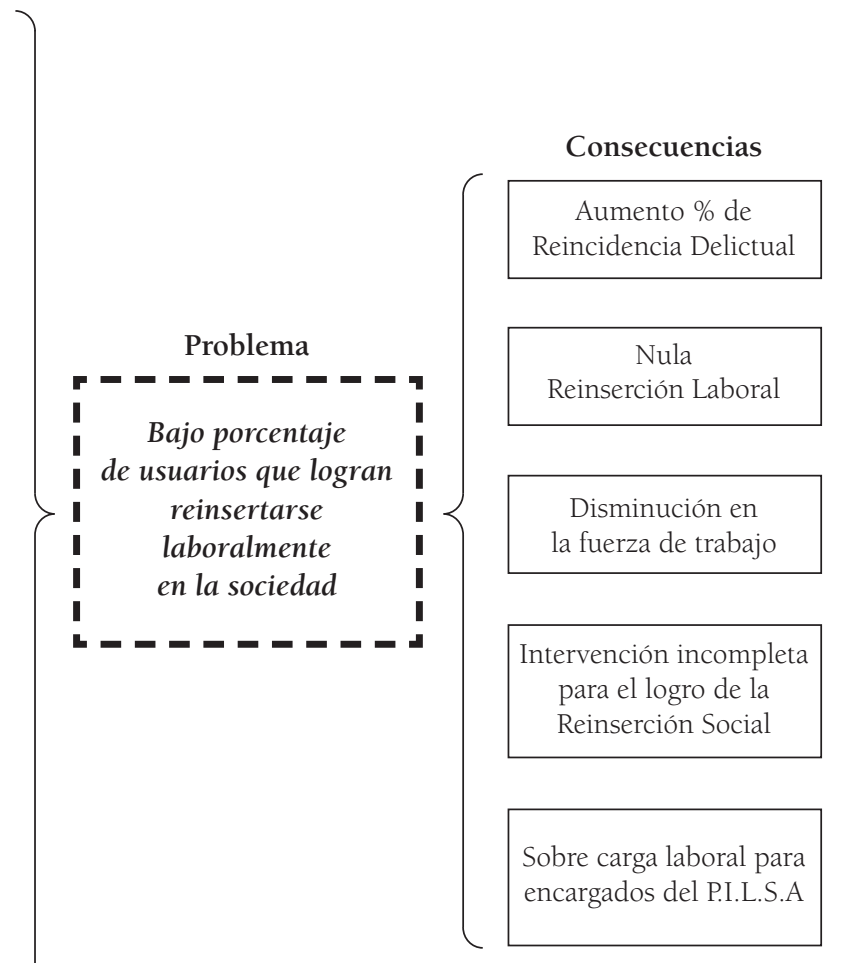
encargados del P.I.L.S.A 


\section{Variables de tratamiento}

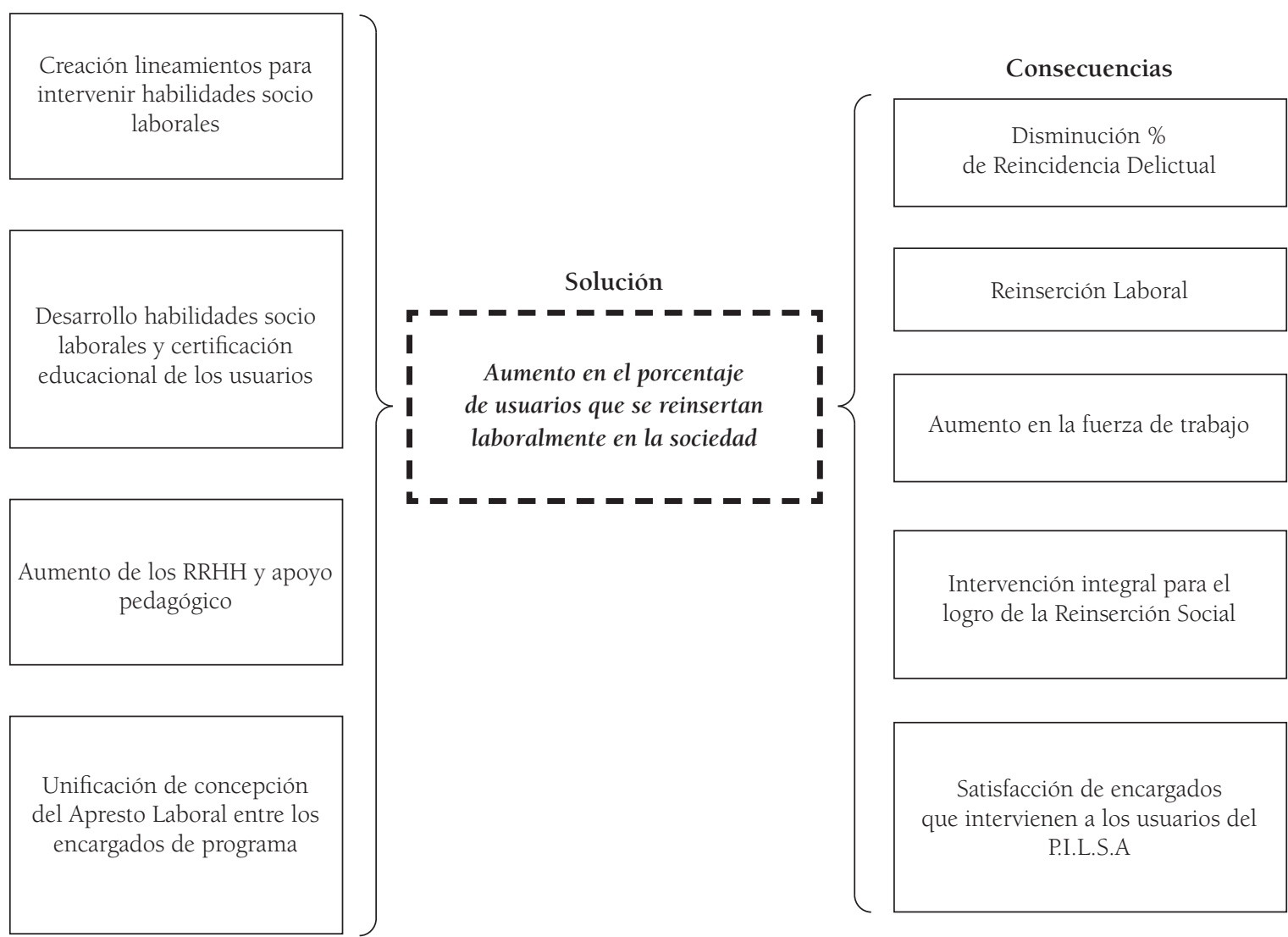

\section{Conclusión}

Los resultados de la investigación diagnóstica realizada con la colaboración de los encargados de los programas a nivel nacional dieron cuenta de tres aspectos principales en la implementación de los talleres de apresto laboral: el primero de ellos se relaciona con la ausencia de lineamientos generales de intervención para todos los PILSA, lo que se traducía en una intervención con contenidos, sesiones y objetivos, que en la mayor parte de los casos no se ajustaba a las necesidades usuarias; el segundo resultado dio cuenta que las principales necesidades sociolaborales de los usuarios, se relacionaban con el déficit de habilidades comunicacionales, habilidades de resolución de conflicto y competencias laborales; finalmente, el tercer resultado del diagnóstico se relaciona con las expectativas para el desarrollo de un futuro taller, el cual -de acuerdo a lo argumentado por los encargados- debería ser un espacio de conocimiento y descubrimiento para los usuarios, donde se desarrollen y potencien sus habilidades, además de entregar información relacionada con el mundo laboral. Estos hallazgos dejan en evidencia el desafío que enfrentan los profesionales encargados de la ejecución del programa, para entregar las herramientas adecuadas para alcanzar la reinserción laboral y social. Considerando que el PILSA es el único programa que trabaja con población que se encuentra cumpliendo condena en el medio libre, se requiere una intervención integral y de calidad para lograr la reinserción laboral y social de sus usuarios.

La necesidad de reinserción laboral y social de las personas que cumplen condena en libertad o poseen antecedentes penales está correctamente identificada y, por lo demás, es ampliamente reconocida y 
exigida por la sociedad. Por otra parte, la disponibilidad de un empleo estable ha sido reconocida como un importante mecanismo de reinserción social (Alós, Martín, Miguélez \& Gibert, 2009). El apego a las normas de la responsabilidad laboral adulta, la posibilidad de sostener una familia y hacerse responsable de otros con ingresos provenientes del trabajo, la capacidad de establecer vínculos sociales significativos en el ámbito laboral y la implantación de una estructura de vida rutinaria y organizada son todas ventajas que han sido ampliamente asociadas con el trabajo estable, especialmente en mercados formales de trabajo (Raphael, 2011). Sin embargo, en nuestro país existe una gran dificultad de reinserción social, así como también escasas oportunidades laborales disponibles para personas que cumplen condena, sobre todo para quienes poseen deficitarias competencias laborales, certificación educativa y experiencia laboral, donde la estigmatización por presentar antecedentes penales dificultan el proceso de reinserción social, lo que evidencia una invisibilización de esta población que no es reconocida por la sociedad. A pesar, de que en nuestro país no existen impedimentos legales para la contratación de exreclusos, la costumbre de solicitar antecedentes legales está muy extendida entre los empleadores. La literatura revisada evidencia que en otros países, los empleadores tienden a no contratar a personas con antecedentes delictuales, por ejemplo cifras de referencia de Estados Unidos; 71\% de los empleadores no contrataría a alguien con antecedentes penales, un $48 \%$ no contrataría a alguien que tuviera más de un año de desempleo, un 59\% a alguien sin experiencia laboral, lo que muestra las enormes barreras de entrada que existen para infractores que acumulan todas estas desventajas. También se ha mostrado que el $60 \%$ de los empleadores siempre chequea los antecedentes delictuales de las personas que contrata (Raphael, 2011).

Es así como las personas que cumplen condena en el medio libre ven obstaculizada la posibilidad de reinsertarse en la sociedad, ya que la integración a la sociedad está definida bajo condiciones específicas, situación que de acuerdo a Kalinsky (2012) obliga al individuo a reorganizarse, sin tener los medios para hacerlo, teniendo que cargar con el estigma de delincuente, lo que genera evidentemente un rechazo y alejamiento de la sociedad. A ello, Pereira (2011) agrega que los procesos de socialización producen cada vez más competencias formales, que progresivamente se desprenden de contenidos concretos, es decir las personas adquieren una identidad abstracta del yo a partir de la expectativa social. A su vez, Le Grand (2003) afirma que la mayor parte de los programas sociales parten de una idealización del sujeto, donde pretenden explicar el comportamiento de una persona y su motivación para actuar mediante una reproducción estática de la realidad, donde las idealizaciones realizan restricciones que captan lo más relevante de la acción humana y la explican de manera apropiada, dejando de lado los diversos grados de vulnerabilidad de un sujeto.

De esta forma, el trabajo se transforma en una herramienta social que no sólo mejora las capacidades para la vida social, sino que también contribuye con el autoestima, ya que el individuo reconoce que su esfuerzo y labor cobra sentido tanto para su familia así como para la comunidad en donde se inserta, por lo que el desempeño de esta actividad lo dignifica y lo diferencia de los demás, condiciones que están estrechamente vinculadas con la reinserción social, en que las características del mercado, de los empleadores y de las personas condicionan el reingreso de estas a la sociedad. Las personas que se encuentran cumpliendo condena en libertad se encuentran sujetas a las condiciones de un mercado laboral exigente y excluyente, el cual se presenta como la única manera de poder reinsertarse a la sociedad, encontrándose en una situación desventajosa en relación a otros miembros de la sociedad, donde la fuente de los conflictos sociales se encuentra en su experiencia, a través del menosprecio o falta de reconocimiento (el maltrato psicológico, la privación de derechos y la desvalorización social) de la sociedad que no les permite acceder igualitariamente a las mismas oportunidades tanto laborales como sociales. Fascioli (2008) sostiene que estas experiencias de injusticia social nacen por la falta de reconocimiento y el logro de este implica un proceso conflictivo que busca cambios sociales tanto institucionales como culturales.

A partir de estos planteamientos y de los resultados de la investigación diagnóstica realizada se sugiere diseñar y ejecutar un taller de habilidades sociolaborales, el cual se encuentre respaldado por las diversas observaciones profesionales realizadas por los encargados de programa, así como también sustentado en las teorías, estudios y experiencias consultadas. Orientando sus contenidos hacia la autonomía, autoconocimiento, desarrollo y potenciación de habilidades sociolaborales de los usuarios, a fin de que cada usuario se reconozca y reconozca al otro como un ciudadano con derechos y deberes, como una forma de invisibilizar la diferencia y de evitar la exclusión. El taller pretende ser uno de los cimientos que, en conjunto con la capa- 
citación y colocación laboral, faciliten la reinserción laboral de los usuarios, y con ello permitirles mediante el acceso a nuevas oportunidades laborales ser valorados y reconocidos por la sociedad. Por ello, para alcanzar esta valoración y reconocimiento es preciso contar con recursos apropiados para efectivamente lograr la reinserción laboral. Si se aseguran las condiciones materiales que permitan superar situaciones adversas, garantizar educación, oportunidades de participación o un ingreso apropiado, se convierten en medios para poder llevar adelante el plan de vida que se considere valioso (Pereira, 2011). Como se mencionó anteriormente, el trabajo desempeña un papel central en el reconocimiento de una persona, ya que son los individuos quienes definen el grado de valoración social que un sujeto puede tener de acuerdo a la actividad que desempeña, y con base en ello tiene la oportunidad de formar su identidad individual, convertirse en un ser autónomo y autorrealizado (Honneth, 2009). Los principales desafíos que se presentan para el desarrollo del taller y el éxito del PILSA se relacionan con la gestión de recursos humanos y económicos ante las autoridades correspondientes para mejorar los procesos de intervención y capacitación del programa. Así como también es preciso destinar recursos para financiar campañas de difusión que apunten a sensibilizar a la población en general, empresarios, organismos sociales, privados y gubernamentales en particular, respecto de las ventajas de las penas sustitutivas a la reclusión y con mayor relevancia del rol del programa laboral, problematizando el rol y responsabilidad que le cabe a toda la sociedad respecto de estas personas en pos de su reinserción laboral y social y de la seguridad pública.

Asimismo, se requiere una mayor coordinación con otros organismos extrainstitucionales, para la suscripción de convenios que permitan a los usuarios acceder a las prestaciones que ofrecen esas instituciones. Estas prestaciones se refieren al financiamiento a trabajadores independientes, asesorías técnicas especializadas o al acceso a colocaciones laborales. Si bien las gestiones que realizan los encargados de programas han generado una red local importante de plazas de trabajo, no son suficientes para cubrir todas las plazas que se requieren para lograr una reinserción laboral de los usuarios. Como ya se mencionó antes, el trabajo que realiza el programa y los encargados es clave, pero debe estar respaldado y apoyado a nivel institucional y, por qué no decirlo, desde el Estado, entidad que debiera generar y garantizar plazas de trabajo para personas que se encuentren cumpliendo condena en todos sus organismos dependientes. De esta forma, empresarios privados, organizaciones con y sin fines de lucro y la sociedad en general podrán visualizar el compromiso social que tiene el Estado con las personas que cumplen condena, de esta forma todos serán coprotagonistas en el proceso de reinserción laboral y social de los usuarios del Programa de Intermediación Laboral en Sistema Abierto de Gendarmería de Chile.

\section{Bibliografía}

- ACEVEdo, A., GUZMÁN, G., PANTOJA, R., \& WEINBORN, C. (2009). Programa de Intervención Psicosocial Especializados en Factores de Riesgo Dinámicos. Manual General del Módulo. Santiago: Gobierno de Chile. Ministerio de Justicia. Gendarmería de Chile.

- AlÓs, R., MARTíN, A., MiguÉlez, F., \& GIBERT, F. (2009). ¿Sirve el trabajo penitenciario para la reinserción?. Un estudio a partir de las opiniones de los presos de las cárceles de Cataluña. Revista Española de Investigaciones Sociológicas (REIS). 127, 11-31.

- ANDREWS, D., BONTA, J., \& WORMITH, S. (2006). The Recent Past and Near Future of Risk and/or Need Assessment. Crime \& Delinquency. 52 (1), 7-27. DOI: 10.1177/0011128705281756.

- ANDREWS, D., GUZZO, L., RAYNOR, P., ROWE, R., RETTINGER, J., BREWS, A., \& WORMITH, S. (2012). Are the Major Risk/Need Factors Predictive of Both Female and Male Reoffendig?: A Test with the eight domains of the level of service/cas management Inventory. Internacional Journal of Offender Therapy and Comparative Criminology. 56 (1), 113-133. DOI: 10.1177/0306624X10395716.

- AÑEZ, M., HAN, P., MORAles, J., \& PÁRRAGA, J. (2008). Asistencia Laboral Penitenciaria y Post Penitenciaria, una propuesta a la reintegración social del recluso. Capítulo Criminológico. 36 (4), 51-88. ISSN:0798-9598.

- ATKIN, C., \& ARMSTrong, G. (2013). Does the concentration of Parolees in a Community Impact Employer Attitudes Toward the Hiring of Ex-Offenders? Criminal Justice Policy Review, 71-93. DOI: 10.1177/0887403411428005.

- Bermejo, J. C., \& MARTíneZ, A. (2006). Motivación e Intervención Social. Cómo trabajar con personas resistentes al cambio. Cantabria: Editorial Sal Terrae Santander

CEPAL (Comisión Económica para América Latina y el Caribe). (2009). Panorama Social de América Latina 2008. Santiago: Naciones Unidas, División de Desarrollo Social y la División de Estadística y Proyecciones Ecoómicas de la CEPAL. 
- CID, J., \& MARTí, J. (2011). El proceso de desistimiento de las personas encarceladas. Obtáculos y Apoyos. Generalitat de Catalunya. Barcelona: Departamento de Justicia. Centro de Estudios Jurídicos y Formación Especializada

- CORBIN, J., \& STRAUSS, A. (2002). Bases de la Investigación Cualitativa: técnicas y procedimientos para desarrollar la teoría fundamentada. Medellín: Universidad de Antioquía. ISBN: 958-655-623-9.

- DAY, A., BRYAN, J., DAVEY, L., \& CASEY, S. (2006). The process of change in offender rehabilitation programmes. Psychology, Crime \& Law, 12:5, 473-487. DOI:10.1080/10683160500151209.

- DiCLEMENTE, C., PROCHASKA, J., \& NORCROSS, J. (1992). In search of how people change: Applications to addictive behaviors. American Psychologist 47 (9), 1102-1114

- DIDIER, M. (1986). Diseño y Evaluación de Programas Sociales. Santiago: Pontificia Universidad Católica de Chile, Escuela de Psicología, Manual de Psicología $\mathrm{N}^{\circ} 1$

- ESPINOZA, A., FERRADA, D., NAVARRO, P., \& VALENZUELA, E. (2012). Informe Final de Evaluación de Programas de Rehabilitación y Reinserción Social. Santiago: Ministerio de Justicia: Gendarmería de Chile.

- ESPINOZA, C., \& VIANO, O. (2008). El desafío de la libertad: Proceso de concesión de beneficios intrapenitenciarios para la reinserción social. Santiago, Chile: Ril Editores.

- FÁbregA, J., MORAleS, A., MUÑOZ, N., \& WELSCH, G. (2012). Reincidencia en el Sistema Penitenciario Chileno. Santiago: Fundación Paz Ciudadana. Universidad Adolfo Ibáñez.

- FASCIOLI, A. (2008). Autonomía y Reconocimiento en Honneth: Un rescate del sistema de eticidad de Hegel en la filosofía contemporánea. Revista Actio 10 , 20-25.

- FLICK, U. (2004). Introducción a la Investigación Cualitativa. Madrid: Morata.

- FUNDACIÓN PAZ CIUDADANA. (2010). Buenas Prácticas en Rehabilitación y Reinserción de infractores de ley. Santiago: Fundación Paz Ciudadana.

- GAllarT, M., \& JACINTO, C. (1995). Competencias Laborales: tema clave en la articulación educación-trabajo. Curso subregional de formación de gerentes de educación técnico-profesional. Montevideo: CINTERFOR.

- GARCÍA, E., GIL, J., \& ROdRíGUEZ, G. (1999). Metodología de la Investigación Cualitativa. Madrid: Ediciones Aljibe

- GENDARMERÍA. (26 de mayo de 2014). Gendarmería de Chile. Obtenido de http://www.gendarmeria.gob.cl/
- GONZÁlEZ, J., \& RAMÍREZ, M. (2008). Reinserción Laboral en el Medio Libre. Desarrollo, Resultados y Desafíos. Revista de Estudios Criminológicos y Penitenciarios. Gendarmería de Chile, 667-702.

- HOllin, C., \& PALMER, E. (2009). Cognitive Skills programmes for offenders. Psychology, Crime \& Law. 15 (2-3), 147-164. DOI: 10.1080/10683160802190871.

- HONNETH, A. (1997). La Lucha por el Reconocimiento, por una gramática moral de los conflictos sociales. Barcelona: Crítica.

- HONneth, A. (2009). Crítica del Agravio Moral. Patologías de la sociedad contemporánea. Buenos Aires: Fondo de Cultura Económica de Argentina.

- HOWARD, P., CLARK, D., \& GARNHAM, N. (2006). An Evaluation of the Offender Assessment System (OASys) in Three Pilots, 1999-2001. OASys Data Evaluation and Analysis Team.

- IBAÑEZ, M. I. (2012). No con un jefe: Las dinámicas de inserción laboral de las personas en situación de calle. Santiago: Universidad Alberto Hurtado.

- JARABA, B., ROMERO, L., \& RUIZ, M. (2005). Competencias Laborales y la formación universitaria. Psicología desde el Caribe. 16, 64-91. ISSN 0123417X.

- KAliNSKY, B. (2012). Condiciones de Cumplimiento de la Libertad Condicional en Argentina. Revista de Derecho 25 (2), 33-58.

- KRAUSE, M. (1995). La investigación cualitativa: un campo de posibilidades y desafíos. Revista Temas de Educación. 7, 19-39. ISSN 0716-7423.

- LAUB, J., \& SAMPSON, R. (2001). Understanding desistance from crime. Crime and Justice. 28, 1-69. DOI: 0192-3234.

- LE GRAND, J. (2003). Motivation, Agency and Public Policy: Of Knights and knaves, Pawns and Queen. New York: Oxford University Press.

- LUONG, D., \& WORMITH, S. (2011). Applying Risk/Need Assement to probation practice an its impact on the Recidivism of Young Offenders. Criminal Justice and Behavior. 38. (12), 1177-1199. DOI: 10.1177/0093854811421596.

- MCMURRAN, M., \& MCCUllOCH, A. (2007). Why don't offenders complete treatment? Prisioner's reasons for non-completion of a cognitve skills programme. Psychology, Crime \& Law, 345-354. ISSN 1068-316X print/ISSN 1477-2744.

- MilKMAN, H., \& WANBERG, K. (2007). CognitiveBehavioral Treatment. A review and Discussion for Corrections Professionals. Washington: U.S. Departament of Justice, National Institute of Corrections.

- MILlER, W., \& ROLLNICK, S. (2002). Motivational Interviewing. Preparing People for Change. New York: The Guilford Press. 
- OlATE, M. (2007). Políticas e Instrumentos de Reinserción Social. Revista de Estudios Criminológicos y Penitenciarios. 11. Ministerio del Interior y Justicia, Subdirección Técnica, Gendarmería de Chile, 7-15.

- OliVERI, K. (2011). Programas de Rehabilitación y Reinserción de los sistemas de cárceles concesionadas y estatales. Santiago, Chile: Sociedad Chile de Políticas Públicas.

- PEREIRA, G. (2010). Reconocimiento y Criterios Normativos. Entrevista a Axel Honneth. Revista Andamios. 7 (13), 323-334.

- PereirA, G. (2011). Justicia Distributiva y Reconocimiento. Un expansión de la propuesta de Honneth. Revista Andamios, 201-232.

- RAPHAEL, S. (2011). Improving employment prospects for former prison inmates. Controling Crime, Strategies and Tradeoffs. Chicago: The University of Chicago Press.

- TARLOW, M. (2008). El rol del empleo en el proceso de reinserción: La experiencia del Centro de Oportunidades para el Empleo (CEO) de Nueva York. Revista de Debates Penitenciarios. 8. Área de Estudios Penitenciarios CESC, 1-26.

- TAYLOR, S., \& BODGAN, R. (1987). Introducción a los Métodos Cualitativos de Investigación. Barcelona: Páidos Ibérica.

- VISHER, C., WINTERFIELD, L., \& COGGESHALL, M. (2005). Ex-offender employment programs and recidivism: A meta-analysis. Journal of Experimental Criminology, 295-317.

- WARD, T., \& MARSHALL, B. (2007). Narrative Identity and Offender Rehabilitation. International Journal of Offender Therapy Comparative Criminology. 51, 279-297.

- WARD, T., \& MARUNA, S. (2007). Rehabilitation. New York: Routledge.

- WHITE, M., \& EPSTON, D. (2004). Medios narrativos para fines terapéuticos. Barcelona: Paidós.

- YAHNER, J., ZWEIG, J., \& REDCROSS, C. (2010). Recidivism Effects of the Center for Employment Opportunities (CEO) Program Vary by Former Prisoners' Risk of Reoffending. New York: MDRC. Building Knowledge to improve Social Policy. 